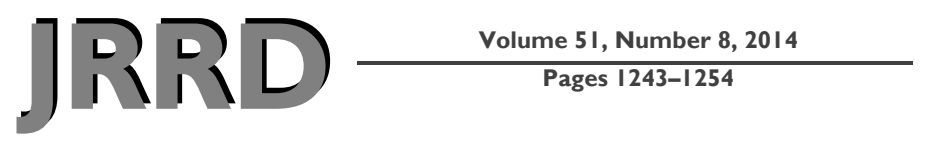

\title{
Symmetrical kinematics does not imply symmetrical kinetics in people with transtibial amputation using cycling model
}

\author{
W. Lee Childers, PhD, CP; ${ }^{*}$ Géza F. Kogler, PhD, $\mathrm{CO}^{2}$ \\ ${ }^{1}$ Cycling Biomechanics Laboratory, School of Applied Physiology, Georgia Institute of Technology, Atlanta, GA; and \\ Department of Prosthetics and Orthotics, Alabama State University, Montgomery, AL; ${ }^{2}$ Clinical Biomechanics Labora- \\ tory, School of Applied Physiology, Georgia Institute of Technology, Atlanta, GA
}

\begin{abstract}
People with amputation move asymmetrically with regard to kinematics (joint angles) and kinetics (joint forces and moments). Clinicians have traditionally sought to minimize kinematic asymmetries, assuming kinetic asymmetries would also be minimized. A cycling model evaluated locomotor asymmetries. Eight individuals with unilateral transtibial amputation pedaled with 172 mm-length crank arms on both sides (control condition) and with the crank arm length shortened to $162 \mathrm{~mm}$ on the amputated side (CRANK condition). Pedaling kinetics and limb kinematics were recorded. Joint kinetics, joint angles (mean and range of motion [ROM]), and pedaling asymmetries were calculated from force pedals and with a motion capture system. A one-way analysis of variance with Tukey post hoc compared kinetics and kinematics across limbs. Statistical significance was set to $p</=0.05$. The CRANK condition reduced hip and knee ROM in the amputated limb compared with the control condition. There were no differences in joint kinematics between the contralateral and amputated limbs during the CRANK condition. Pedaling asymmetries did not differ and were $23.0 \%+/-9.8 \%$ and $23.2 \%+/-12.0 \%$ for the control and CRANK conditions, respectively. Our results suggest that minimizing kinematic asymmetries does not relate to kinetic asymmetries as clinically assumed. We propose that future research should concentrate on defining acceptable asymmetry.
\end{abstract}

Key words: artificial limb, asymmetry, biomechanics, cycling, gait analysis, joint kinetics, kinematics, lower-limb amputation, prosthesis, rehabilitation.

\section{INTRODUCTION}

The complex task of human locomotion involves integration of the neuromuscular and musculoskeletal systems, taking into account the individual's underlying morphology, the environment, and specific task demands to move the body in a stable and controlled manner. A person with a unilateral transtibial amputation (TTA) loses a significant portion of his or her motor system, e.g., the foot and ankle joint and a portion of the shank, and relies on the prosthesis as substitutive replacement for locomotion. This restorative human and prosthesis system is inherently asymmetric and induces considerable motor asymmetries during locomotor tasks [1-5]. Kinetic asymmetries, i.e., higher joint loading on the contralateral limb, have been implicated as a causal factor in the higher incidence of knee osteoarthritis in the contralateral limb [6]. Although prosthetists are aware of the implications of kinetic asymmetries on an individual with an amputation, the typical clinical setting is not conducive to measuring

\footnotetext{
Abbreviations: ASIS $=$ anterior superior iliac spine, $\mathrm{ROM}=$ range of motion, TTA $=$ transtibial amputation.

* Address all correspondence to W. Lee Childers, PhD, CP; Alabama State University, PO Box 271, Montgomery, AL 36101-0271; 334-229-8808; fax: 334-229-5878.

Email: Ichilders@alasu.edu

http://dx.doi.org/10.1682/JRRD.2013.11.0241
} 
joint loading. Therefore, the link between kinetic and kinematic asymmetries and their potential clinical applicability is of considerable interest.

Locomotor symmetry is often assumed as a prosthetic performance goal during rehabilitation [3,7-9]. Prosthetists typically seek to optimize the movements of persons with amputation by configuring prosthetic alignment, i.e., adjusting task mechanics, to promote symmetrical joint kinematics and presumably more symmetrical joint kinetics during gait [9]. The prosthetist traditionally relies on observational gait analysis to interpret limb kinematics with clinical supposition that kinematic and kinetic asymmetries are associated [9]. Newer technologies that allow for direct measurement of prosthetic loading in a clinical setting have enormous promise to improve clinical outcomes [10-13]. They have, as of yet, not superseded the traditional method of observational gait analysis during normal clinical practice.

The clinical assumption that prosthetic alignment alters limb kinematics and kinetic asymmetries suggests a link between these variables; however, the mechanism is not well understood [3]. Some reports suggest asymmetry in ground reaction forces are related to biasing the body's center of mass toward the contralateral limb [14-15]. Indeed, the center of mass position is dictated by limb kinematics [16-17], and this creates one link between limb kinematics and kinetics. However, other factors produce interlimb asymmetries, e.g., interactions between the limb and socket interface [3,5], loss of sensorimotor input [4], altered musculoskeletal geometry [1,5], and loss of propulsion due to the lack of ankle plantarflexors $[2,4,13]$. These other factors may be more related to how the human and prosthesis system is used for the propulsive task of gait. These additional propulsive influences may have greater relevance than the relationship between center of mass and ground reaction forces.

Successful gait requires the body to perform balance and propulsion tasks simultaneously. These dual functions blur relationships between kinematic and kinetic asymmetries during gait. Cycling is a rhythmic motor task similar to walking yet has the methodological advantage of isolating the locomotive propulsion by supporting the body via the saddle, allowing the motor system to focus on energizing the bicycle [18]. In both cycling and walking, the motor system utilizes similar neural centers [19], coordinates muscle activity in accordance with their respective functional roles [20], utilizes similar optimization strategy [21], and is a well-used model to study motor system behavior during rhythmic locomotion [5,18-24]. The cycling task also provides a method to control lower-limb kinematics by altering the geometric constraints between the rider and the bicycle, e.g., rider position [18]. Lower-limb kinematics are not symmetrical during amputee cycling because the prosthetic ankle is fixed while the contralateral ankle extends through the bottom of the pedal stroke and flexes through the top. The lack of ankle motion in the amputated limb leads to greater knee and hip flexion and extension to complete the pedal stroke. Normal ankle joint range of motion (ROM) during pedaling is about $20^{\circ}$ as it extends (plantarflexes) during the bottom and flexes (dorsiflexes) through the top of the pedal stroke [25]. Limiting ankle motion on one limb, e.g., a prosthesis, alters the mechanics of cycling by increasing the ROM of the hip and knee joints to compensate for ankle motion losses [26].

Shortening the crank arm on the amputated limb is a method to adjust bicycle geometry and minimize kinematic asymmetries and has been shown to reduce kinetic pedaling asymmetries in a small number of cyclists with TTA (2 out of 3) [26].

Pedaling asymmetries have been reported in force and work generation about the crank spindle [5]. Force asymmetries during cycling quantify the difference between the total amount of force generated in each leg and are related to strength and/or inertial differences between limbs, i.e., fixed constraints between conditions [27]. Work asymmetry quantifies the difference in torque generated between limbs [27]. The presence of work asymmetry indicates a deficiency in propulsion between the two limbs. Force asymmetries are generally constant within a person, whereas work asymmetries depend on task mechanics [5,28].

The purpose of this research was to define the relationship between kinematic and kinetic asymmetries during a propulsive task, i.e., stationary cycling. We hypothesized that (1) the shortened crank arm would produce symmetrical limb kinematics, (2) force asymmetry would remain constant, (3) work asymmetry would be reduced with a reduction in kinematic asymmetries, and (4) motor system compensation to the shortened crank would be demonstrated as a change in joint moments at the knee and hip. The rationale for hypothesis 3 was that the shortened crank arm may normalize cycling task mechanics between the two limbs and therefore may be an influential factor on work asymmetry (how each limb contributes to total work). 


\section{METHODS}

A group of eight male recreational cyclists with TTA (body mass [mean \pm standard deviation]: $81.3 \pm 16.1 \mathrm{~kg}$, height: $1.84 \pm 0.09 \mathrm{~m}$, age: $33.7 \pm 10.0 \mathrm{yr}$ ) were recruited. Inclusion criteria were having a TTA secondary to trauma or cancer, being a prosthetic limb user for $\geq 1 \mathrm{yr}$, regularly riding a bicycle, and self-reporting cardiovascular exercise at least $6 \mathrm{~h} / \mathrm{wk}$. The inclusion criteria were based on health screening protocols to minimize cardiovascular risk [29]. Exclusion criteria included having any secondary neuromuscular complications, cardiovascular disease, diabetes, peripheral vascular disease, or anything that would limit exercise ability.

Volunteers pedaled a stationary electromagnetically braked ergometer (Excalibur Sport, Lode BV; Groningen, the Netherlands) adapted with a custom-fabricated pedal and crank system. Constant resistance was set at $15 \mathrm{Nm}$, and volunteers controlled cadence at 90 rotations $/ \mathrm{min}$ based on a handlebar-mounted tachometer. The combination of workload and cadence meant power output was $\sim 150 \mathrm{~W}$. This is a common load and cadence combination in motor control literature using cycling [18]. The baseline condition was with the crank arm lengths set to $172 \mathrm{~mm}$ (control condition), which is typical for road cycling, while the experimental condition had the crank arm length on the amputated side set at $162 \mathrm{~mm}$ (CRANK condition). Shortening the crank on the amputated side reduces the geometric asymmetries between the two lower limbs of an individual with TTA. The ankle in the contralateral limb actively plantarflexes at the bottom of the pedal stroke and dorsiflexes at the top, giving a total ROM of $\sim 20^{\circ}$ [25]. Using average human anthropometrics, this means the shank and ankle (with the cycling cleat at the base of the first metatarsal) are effectively lengthening and shortening a total of $19.8 \mathrm{~mm}$ [30]. Therefore, shortening the crank $10 \mathrm{~mm}$ on the amputated side (half the linear ROM) brings the pedal closer at the bottom and farther away at the top of the stroke to decrease kinematic asymmetries and offers a method to account for ankle joint movement of the contralateral limb [26]. The linear ROM of the crank spindle relative to the ankle joint marker was calculated on the contralateral limb during cycling to verify whether shortening the crank arm $10 \mathrm{~mm}$ was sufficient for these subjects.

Volunteers were given a 5 to $10 \mathrm{~min}$ warm-up period at $75 \mathrm{~W}$ and self-selected cadence. The subject's pre- dicted maximal heart rate was determined [29]. A heart rate monitor (Polar CS400, Polar Electro Oy; Kempele, Finland) was worn during all trials to verify submaximal workload and minimize fatigue effects. Data were collected $30 \mathrm{~s}$ after the volunteers achieved and maintained steady-state cadence for at least $30 \mathrm{~s}$.

The pedal contained dual piezoelectric elements to measure forces normal and orthogonal to the pedal surface [31] adapted with a commercial "clipless" pedal system (Figure 1) [32]. Saddle height was initially set to 98 percent leg length, and then fine adjustments $( \pm 1 \mathrm{~cm})$ were

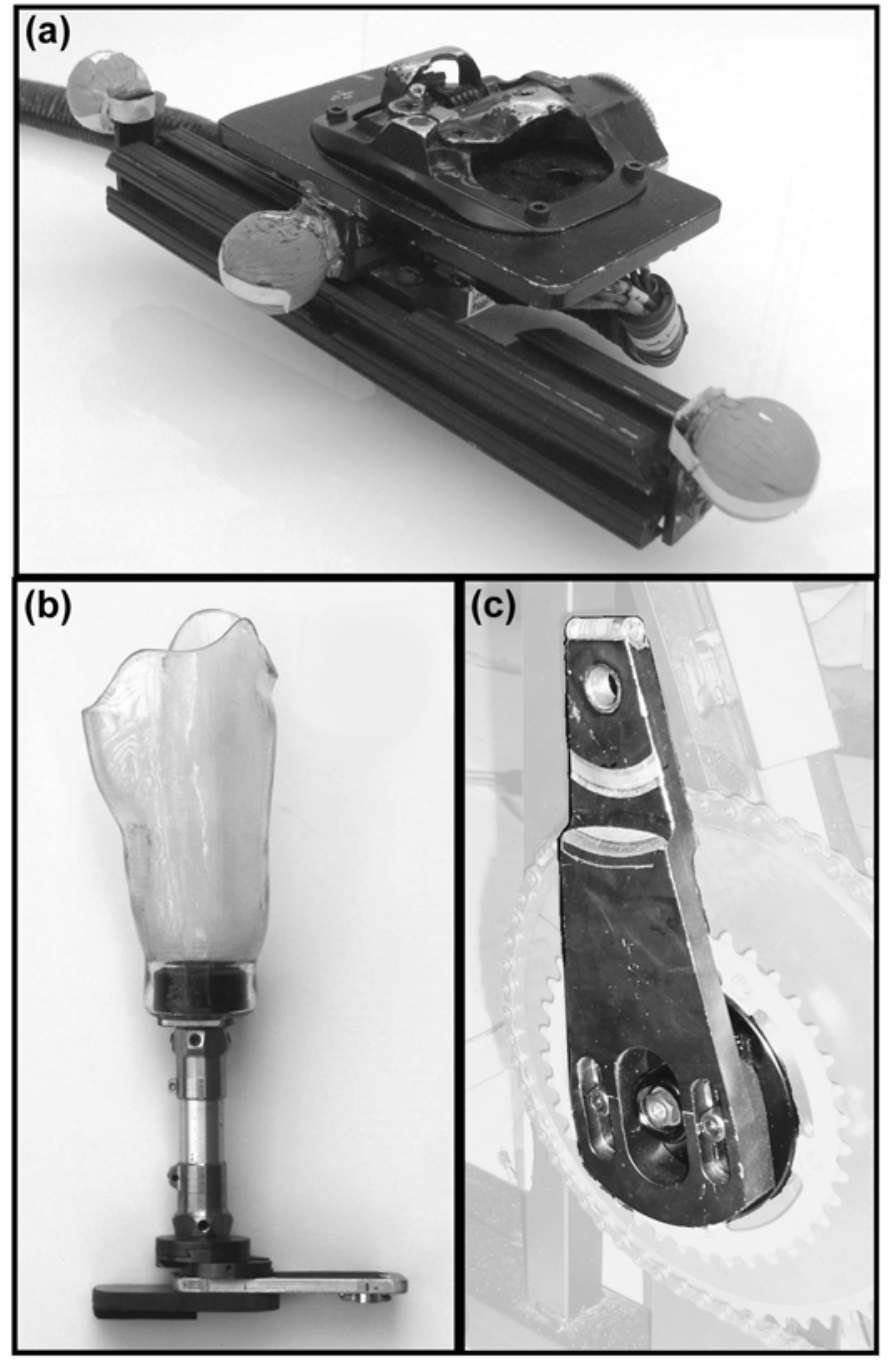

Figure 1.

(a) Instrumented pedal with cleated pedal interface, (b) example of prosthetic socket and stiff aluminum foot used in experiment, and (c) adjustable crank arms used for experiment. 
made for comfort [26]. The handlebar reach, drop, and seat tube angle were adjusted to the subject's position as measured from his primary bicycle, or (if his bicycle was unavailable) the position was based on established bicycle positioning protocol [26,33]. Top dead center of the crank cycle is defined as when the crank arm is vertical.

The crank arms were custom machined from aluminum and specialized to this pedal system (Figure 1). The crank arms consisted of two pieces: crank hub and crank arm. The crank hubs were machined to fit a standard square taper bottom bracket and a gear-driven potentiometer necessary to calculate crank position. The crank arm was slotted to allow the researcher to alter crank arm length by loosening two recessed socket head cap screws, sliding the crank arm relative to the crank hub, and then retightening the screws. The resulting crank hub and arm system allowed for crank arm lengths between 162 and $185 \mathrm{~mm}$ (172 and $162 \mathrm{~mm}$ used for this experiment). In addition, the crank arms were machined to offset the pedal bodies toward the midline of the cycle ergometer. This was necessary because the design of the piezoelectric force pedal bodies offset the center of the pedal laterally and would increase pedal width if not taken into account. The combination of the medial offset incorporated in the crank arm design as well as machining and positioning of the pedal interface achieved a pedal width within conventional road bicycle specifications.

Prosthetic stiffness, e.g., prosthetic foot, and prosthetic alignment was controlled. A rigid $155 \times 50 \times 10 \mathrm{~mm}$ plate of 6061-T6 aluminum served as the "prosthetic foot" because a previous study demonstrated that pedaling asymmetries and pedal force variability were minimized using this foot (Figure 1) [5]. The subject's prosthetic socket was duplicated with an electromagnetic shapecapturing device (TracerCAD, The Ohio Willow Wood Company; Columbus, Ohio) and a thermoplastic prosthetic socket replica produced by a central fabrication facility (Prosthetic Design Inc; Clayton, Ohio). A portion of the lateral wall of the prosthetic socket was removed, allowing for knee center marker placement. Prosthetic suspension was controlled across volunteers and included a silicon liner with mechanical pin type suspension (X-PSHPLUS, Prosthetic Design Inc). This type of prosthetic suspension attached the distal end of the residual limb to the base of the prosthetic socket via a pin and lock device, effectively creating a pseudojoint. The cycling cleat was mounted in the approximate location of the first metatarsal head in the sagittal plane and the center of the foot in the frontal plane. The socket medial and lateral alignment relative to the foot was transferred from the subject's personal prosthesis.

Analog data (force transducers and crank position) were collected at $300 \mathrm{~Hz}$. Kinematic data were collected at $60 \mathrm{~Hz}$ using a motion capture system (Peak Performance, Vicon Motion System Inc; Oxford, United Kingdom) and digitized using Peak Performance software. An electronic pulse synchronized force and video records. Reflective markers were placed on the subject over the sacrum as well as bilaterally over the greater trochanter of the femur, anterior superior iliac spine (ASIS), lateral epicondyle of the femur, and lateral malleolus. The proximal lateral wall of the prosthesis was removed to allow placement of the knee marker, and there was an additional marker placed on the prosthesis at the location of the distal residuum. This allowed for calculation of the angular displacement between the residual limb in the prosthesis because the distal residuum pivots around the location of the prosthetic lock and the knee marker is directly over the anatomical knee joint [34]. This allowed joint kinematic calculations to be tied to physiological limb segments and account for any angular movement between the residuum and prosthetic socket. A static calibration trial was performed when the subject initially mounted the ergometer. The subject was asked to be still with the crank parallel to the ground for $10 \mathrm{~s}$ while data were collected. This information was used later to calculate the center of hip joint rotation based on the ASIS marker [35].

Kinetic and kinematic data were processed in customwritten MATLAB software (MATLAB, MathWorks; Natick, Massachusetts). The kinetic data were digitally filtered using a fourth-order zero-lag Butterworth filter with a $15 \mathrm{~Hz}$ cutoff frequency. The kinematic coordinate data were smoothed using a Woltring quintic spline. The kinetic and kinematic data of eight complete crank cycles were time normalized to 100 data points and averaged together.

The ankle joint center of rotation was calculated based on equations from Vaughan et al. [36]. The knee joint center of rotation was calculated based on data presented by Smidt that related actual knee joint center to the lateral epicondyle of the femur [37]. Limb segment center of mass location, mass, and moment of inertia in the sagittal plane were calculated from regression equations [38] and modified for the appropriate joint centers [39]. Properties of the residual limb and prosthesis were calculated using a pendulum method [40-41]. Joint moments were calculated using equations in inverse dynamics for the sagittal plane 
[42]. Included joint angles were used to represent joint kinematics for the knee and ankle, whereas thigh position relative to the horizontal defined hip joint angle (limb schematic in Figure 2). Average and ROM of limb kinematics were calculated for analysis.

A one-way analysis of variance evaluated differences in joint kinematic and joint kinetic variables between the contralateral and amputated limbs across the control and CRANK conditions (total of four groups: contralateral limb and control condition, contralateral limb and CRANK condition, amputated limb and control condition, and amputated limb and CRANK condition). When statistical significance was found, a Tukey post hoc analysis revealed specific differences between groups. Paired $t$-tests were used to evaluate differences in force and work asymmetries across the control and CRANK conditions. Statistical significance was set at $p<0.05$.

\section{RESULTS}

Significant differences were observed in the average knee angle and hip ROM between the contralateral and amputated limbs in the control condition, indicating that these volunteers were kinematically asymmetrical before altering crank arm length (Figure 2). The CRANK condition significantly reduced the knee and hip ROM in the amputated limb compared with the control condition (Figure 2). No significant differences were found between the contralateral and amputated limbs within the CRANK condition, indicating that the shortened crank arm did minimize kinematic asymmetry (Figure 2). No statistically significant differences were noted in contralateral limb ankle, knee, and hip kinematics between the control and CRANK conditions. The linear ROM of the crank spindle relative to the ankle joint center of the contralateral limb was $23.9 \pm$ $3.4 \mathrm{~mm}$, calculated to verify that the CRANK condition selected was appropriate for these subjects. Therefore, the "ideal" amount to shorten the crank was $12 \mathrm{~mm}$ or about $2 \mathrm{~mm}$ more than what was done for this experiment.

Pedaling asymmetries with regard to work and force generated at the crank were not significantly different between the control and CRANK conditions ( $p=0.85$ and 0.56, respectfully) (Figure 3). Work asymmetry decreased a small amount in four volunteers and increased in the other four volunteers that used the shortened crank (Figure 4). The differences in work asymmetry between conditions were small within volunteers and
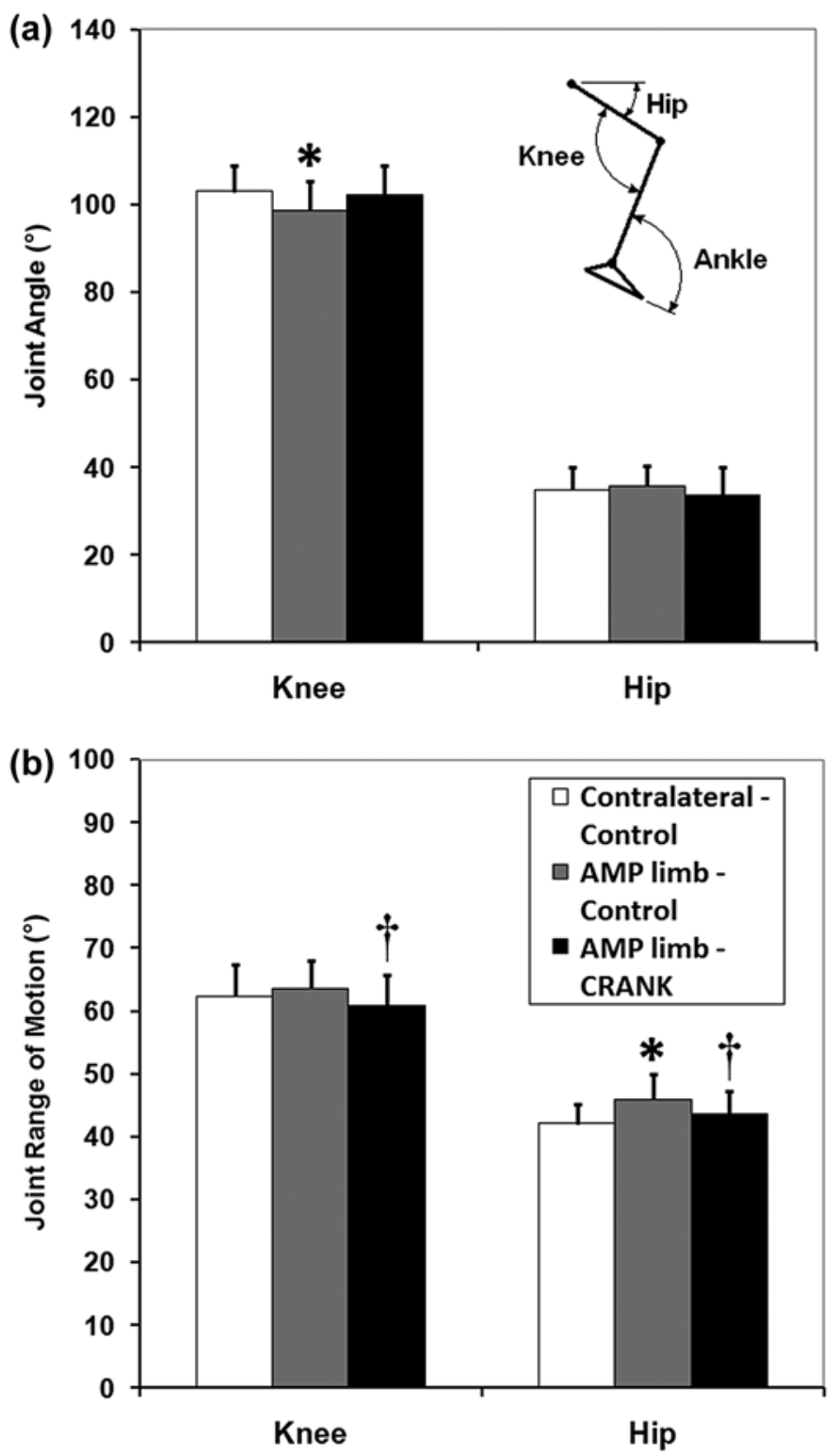

Figure 2.

Average (a) joint angle and (b) range of motion for knee and hip joint. Information for contralateral limb during CRANK condition is not shown for clarity because there were no differences in contralateral limb kinematics between control and CRANK conditions. *Statistically significant difference from contralateral limb within condition. ${ }^{\dagger}$ Statistically significant difference from control condition within amputated (AMP) limb.

generally within normal variability (Figure 4) [27]. Crank torque production was similar in the amputated limb between conditions (Figure 5). 


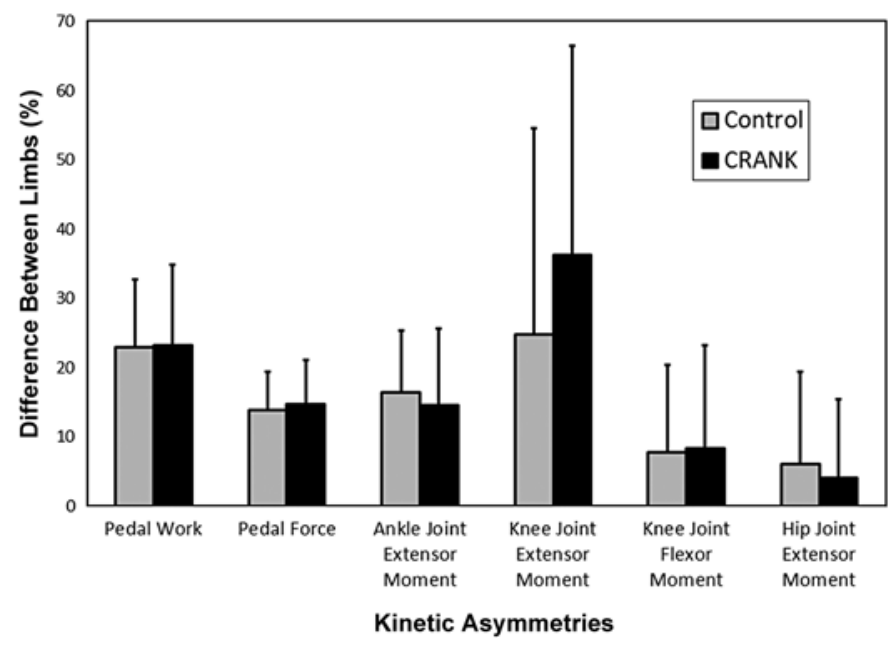

Figure 3.

Pedaling asymmetries regarding work produced about crank spindle (work asymmetry) as well as force produced at pedal (force asymmetry) and asymmetries in average joint moments. No significant differences exist in pedaling asymmetries between control and CRANK conditions.

The ankle joint moment demonstrated significant asymmetry between the contralateral and amputated limbs during both the control and CRANK conditions regarding the average ( $p=0.004)$ (Table 1) and peak $(p=0.005)$ (Table 2) extension moment but showed no changes comparing across control and CRANK conditions within the amputated limb ( $p=0.84$ and 0.44 , respectfully).

A significant asymmetry was observed for the knee joint moment between the contralateral and amputated limbs during both the control and CRANK conditions with regard to the average ( $p=0.004)$ (Table 1) and the peak extension moment $(p=0.04)$ but not with the flexor moment $(p=0.10)$ (Table 2). The average and peak knee joint extensor moment was significantly reduced from the control to the CRANK condition within the amputated limb $(p=0.04)$ (Tables 1-2). The reduced knee extensor moment within the amputated limb during the CRANK condition occurred in all eight volunteers regardless of whether the individual subject demonstrated a change in work asymmetry at the crank, thereby increasing asymmetries between the two limbs despite having symmetrical kinematics.

The average (Table 1) and peak magnitude (Table 2) hip extensor moment did not show any significant differences between the control and CRANK conditions ( $p=0.13$ and 0.12 , respectfully). However, the CRANK condition

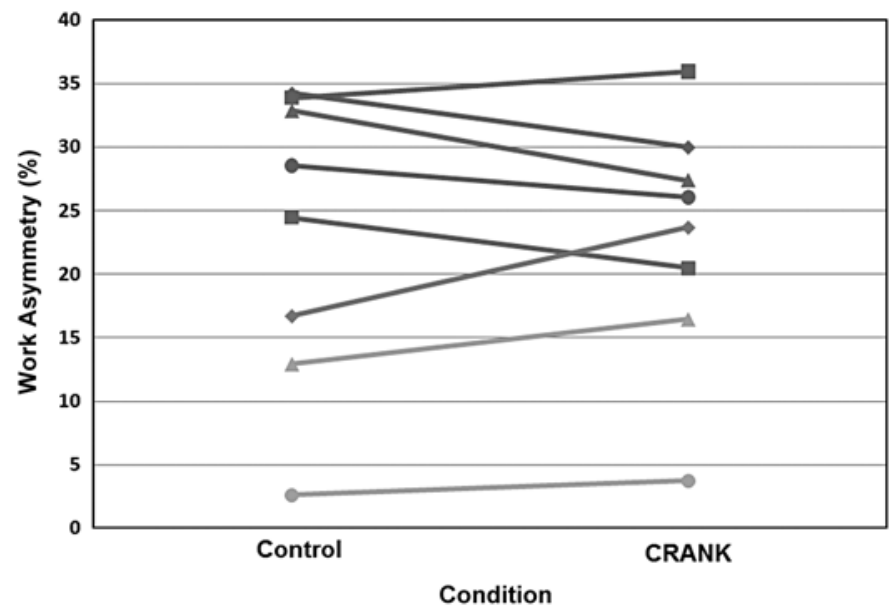

Figure 4.

Change in work asymmetry between conditions demonstrating four subjects with decreased and four subjects with increased asymmetry with shortened crank. Each line represents data for individual subject.

demonstrated a trend (but not significant: $p=0.12$ ) toward a greater hip extensor moment when the knee moment was reduced $\left(\sim 60^{\circ}-120^{\circ}\right)$ in the amputated limb, thereby demonstrating increased asymmetry between contralateral and amputated limbs during the CRANK condition (Figure 6).

In summary, shortening the crank arm on the amputated side removed the significant differences between the contralateral and amputated limbs regarding limb kinematics (average included joint angle and ROM) resulting in kinematic symmetry. However, there remained significant kinetic asymmetries (work and force production at the pedal and in joint moments) between the contralateral and amputated limbs during the control condition, and those asymmetries remained during the CRANK condition. The contralateral limb did not alter crank torque or joint moments between the control and CRANK conditions. The amputated limb did demonstrate a significant reduction in knee joint moment and a trend toward increasing hip joint moment between the control and CRANK conditions, thereby increasing the already present joint moment asymmetries.

\section{DISCUSSION}

One of the most important findings was that utilizing a shortened crank on the amputated side minimized kinematic asymmetries (limb segment angles) but had no significant 


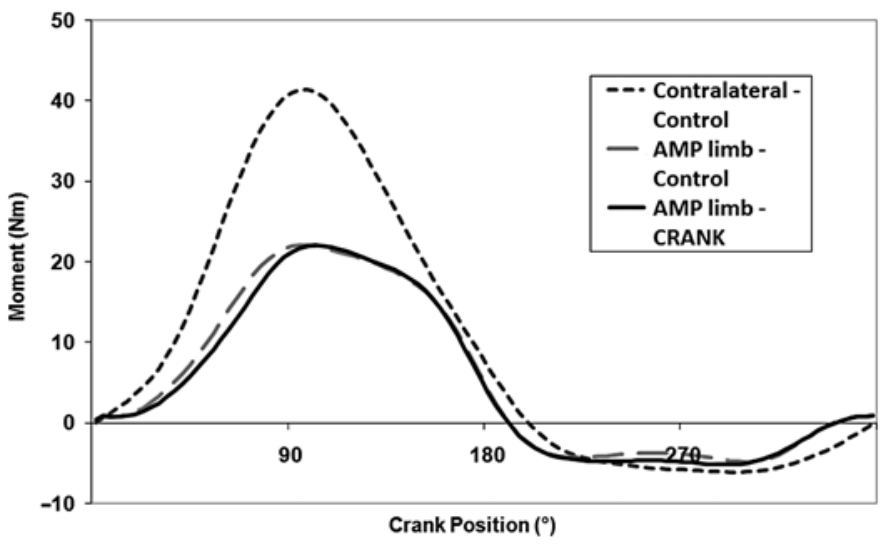

Figure 5.

Crank torque for contralateral limb during control condition (short dashed line), amputated (AMP) limb during control condition (long dashed line), and AMP limb during CRANK condition (solid line).

effect on work or force asymmetries. This observation indicates that the amputated limb performed an equal amount of work about the crank spindle in both conditions. Force asymmetry is related to strength and/or inertial imbalances between limbs [27]. These asymmetries are "fixed" within an experiment and should not change. The force asymmetries data were also similar to a prior study on a separate group of cyclists with TTA, suggesting that these data are representative of a larger sample and instrumentation error was minimized [5]. The stated hypothesis 2 was accepted and hypothesis 3 was rejected because limb kinematics did not have an effect on pedaling kinetics (force asymmetry, work asymmetry, and crank torque). The differences in work asymmetry within a subject were still relatively small (mean absolute difference: 3.7\%) and thus within the normal intersubject variability for this measure [43]. In partial contrast, prior research showed that two out of three cyclists with TTA reduced work asymmetries with a shortened crank arm ( 10\%), while the third subject experienced no change [26]. The difference may be explained by results from individual data, indicating a 50/50 chance of selecting a subject that would demonstrate reduced asymmetry with a shorter crank (Figure 5). Comparing the results with past pilot work demonstrates the necessity of taking larger data sets to fully understand the intervention effects on prosthetic performance before publishing [26].

Shortening the crank arm on the amputated side did produce symmetrical joint motion between limbs by demonstrating (1) no statistical differences between the contralateral and amputated limbs regarding mean joint angle or ROM during the CRANK condition and (2) a statistical difference between the control and CRANK conditions within the amputated limb (Figure 2). The linear ROM of the crank spindle relative to the ankle joint center of the contralateral limb was calculated to verify that the CRANK condition selected was appropriate for these subjects. Those results demonstrated a linear ROM of $23.9 \pm$ $3.4 \mathrm{~mm}$, meaning the "ideal" amount to shorten the crank was $12 \mathrm{~mm}$ and is close to the $10 \mathrm{~mm}$ used in this experiment that still produced a measureable change in limb kinematics. This method allowed for the subject to locomote with symmetrical kinematics without providing any visual feedback or cueing associated with gait studies investigating interlimb symmetry and thereby minimizing supraspinal input into the motor system [44].

The combination of joint moments used to deliver loads to the crank between the contralateral and amputated limbs remained significantly different (asymmetric) between the control and CRANK conditions. The contralateral limb did not alter kinetic output (joint moments or crank torque) between the control and CRANK conditions. Therefore, significant asymmetries remained (in fact, worsened) between the contralateral and amputated limbs at each joint and at the pedal despite having symmetrical kinematics.

Table 1.

Joint moment (mean \pm 1 standard deviation) averaged over crank cycle.

\begin{tabular}{|c|c|c|c|c|}
\hline Limb and Condition & $\begin{array}{l}\text { Ankle Extensor } \\
\text { Moment (Nm) }\end{array}$ & $\begin{array}{l}\text { Knee Extensor } \\
\text { Moment (Nm) }\end{array}$ & $\begin{array}{c}\text { Knee Flexor } \\
\text { Moment (Nm) }\end{array}$ & $\begin{array}{l}\text { Hip Extensor } \\
\text { Moment (Nm) }\end{array}$ \\
\hline \multicolumn{5}{|l|}{ Contralateral Limb } \\
\hline Control & $19.0 \pm 3.5$ & $16.0 \pm 5.5$ & $17.0 \pm 4.5$ & $39.0 \pm 6.5$ \\
\hline CRANK & $18.0 \pm 4.3$ & $17.0 \pm 5.3$ & $18.0 \pm 4.6$ & $41.0 \pm 8.0$ \\
\hline \multicolumn{5}{|l|}{ Amputated Limb } \\
\hline Control & $13.0 \pm 2.5^{*}$ & $9.9 \pm 4.0^{*}$ & $15.0 \pm 3.4$ & $35.0 \pm 8.2$ \\
\hline CRANK & $13.0 \pm 3.2^{*}$ & $7.4 \pm 3.9^{* \dagger}$ & $15.0 \pm 3.7$ & $38.0 \pm 9.2$ \\
\hline
\end{tabular}


JRRD, Volume 51, Number 8, 2014

Table 2.

Peak joint moment (mean \pm 1 standard deviation) within crank cycle.

\begin{tabular}{lcccc}
\hline Limb and Condition & $\begin{array}{c}\text { Ankle Extensor } \\
\text { Moment (Nm) }\end{array}$ & $\begin{array}{c}\text { Knee Extensor } \\
\text { Moment (Nm) }\end{array}$ & $\begin{array}{c}\text { Knee Flexor } \\
\text { Moment (Nm) }\end{array}$ & $\begin{array}{c}\text { Hip Extensor } \\
\text { Moment (Nm) }\end{array}$ \\
\hline $\begin{array}{l}\text { Contralateral Limb } \\
\text { Control }\end{array}$ & $45.0 \pm 11.0$ & $33.0 \pm 13.0$ & $37.0 \pm 10.0$ & $82.0 \pm 20.0$ \\
CRANK & $43.0 \pm 9.9$ & $32.0 \pm 11.0$ & $40.0 \pm 10.0$ & $88.0 \pm 24.0$ \\
Amputated Limb & $34.0 \pm 8.8^{*}$ & $18.0 \pm 7.2^{*}$ & $35.0 \pm 11.0$ & $69.0 \pm 23.0$ \\
$\quad$ Control & $34.0 \pm 11.0^{*}$ & $14.0 \pm 8.0^{* \dagger}$ & $35.0 \pm 12.0$ & $76.0 \pm 26.0$ \\
CRANK & & & \\
${ }^{*}$ Statistically significant difference from contralateral limb within condition. & \\
${ }^{\dagger}$ Statistically significant difference from control condition within amputated limb. & \\
\hline \hline
\end{tabular}

There were some notable changes within the amputated limb regarding knee and hip joint moments between the control and CRANK conditions (Figure 6). The reduced knee extensor moment was associated with an increase in the hip extensor moment with no change in the ankle joint moment within the amputated limb from $60^{\circ}$ to $120^{\circ}$ of crank rotation (Figure 6). These data indicate that the hip and knee joint compensated to maintain total output seen as no change in the ankle moment and crank torque (Figures 5-6). We speculate that the combined changes in knee and hip joint moments may be needed to maintain torque output with a shortened crank arm. A shortened crank arm typically requires an increase in force normal to the crank arm during the power phase $\left(45^{\circ}-135^{\circ}\right)$ to maintain crank torque. Gregor et al. stated that an increased force during this region, given the orientation of the limbs, will produce an increase in the hip extensor moment and decrease in the knee extensor moment, thereby increasing force output to compensate for the shorter crank and maintain crank torque [45]. Therefore, the joint kinetics represent intralimb changes in how the participants adapted to utilize a shortened crank arm rather than a change in total limb output, further explaining why there was no change in work asymmetry.

Cycling is a constrained motor task in which limb kinematics may be manipulated by changing the position of the rider. Walking, a less constrained form of locomotion, includes a phase when the foot is not in contact with the ground. When the foot is not in contact with the ground, prosthetic users can alter step length and timing [45]. Prosthetic users also demonstrate asymmetries in ground reaction forces and joint kinetics [1-4], and these asymmetries have been suggested as an underlying factor in the higher prevalence of osteoarthritis in the contralateral limb [6]. Our study selected the constrained cycling task because it allowed us to minimize kinematic asymmetries and focus on the propulsive aspects of locomotion while utilizing a rhythmic motor task and minimize supraspinal influences [18-19,22-24]. Regardless of the constrained or unconstrained nature of these two locomotor tasks, kinematic and kinetic asymmetries have been documented in both [1-5,7-9,11,44], and this research demonstrated that significant kinetic asymmetries remain, despite symmetrical limb movement indicating that kinetic asymmetries may be more related to differences in the propulsive ability of each limb.

\section{CONCLUSIONS}

In conclusion, the results show a limited relationship between kinematic and kinetic asymmetries as supported in earlier reports [1]. Cycling was used as the locomotor task because it is predominantly a propulsive task, whereas gait is a dual balance and propulsive task. The finding refutes the common prosthetic and rehabilitative goal to minimize kinematic asymmetries in the belief that this will also minimize kinetic asymmetries [9]. These results indicate kinetic symmetry may not be a practical realization in persons with unilateral TTA even in the presence of kinematic symmetry. Task performance appears to be a more heavily weighted controller than task symmetry. This notion is supported by Winter and Sienko: "Any human system with major structural asymmetries in the neuromuscular and musculoskeletal systems cannot be optimal when the gait is symmetrical. Rather, a new nonsymmetrical optimal is probably being sought by the amputee within the constraints of his residual limb and the mechanics of his prosthesis" [1]. Task mechanics have been shown to heavily influence motor behavior $[1,18-24,45]$, and in the case of a person with a TTA, task mechanics have been substantially altered. In this regard, 
optimization of task mechanics (socket design, prosthetic foot mechanical properties and alignment, etc.), given the physiological constraints of the person (neuromusculoskeletal system losses), may improve locomotor perfor-

(a)

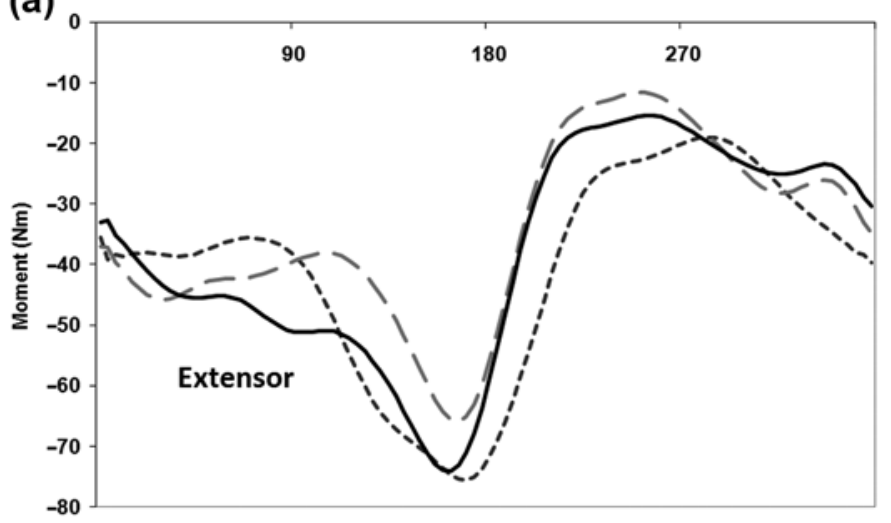

(b)

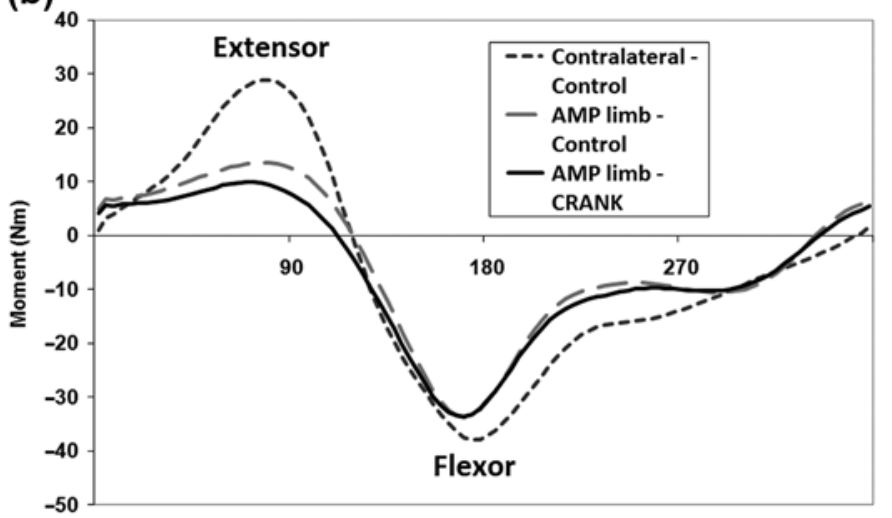

(c)

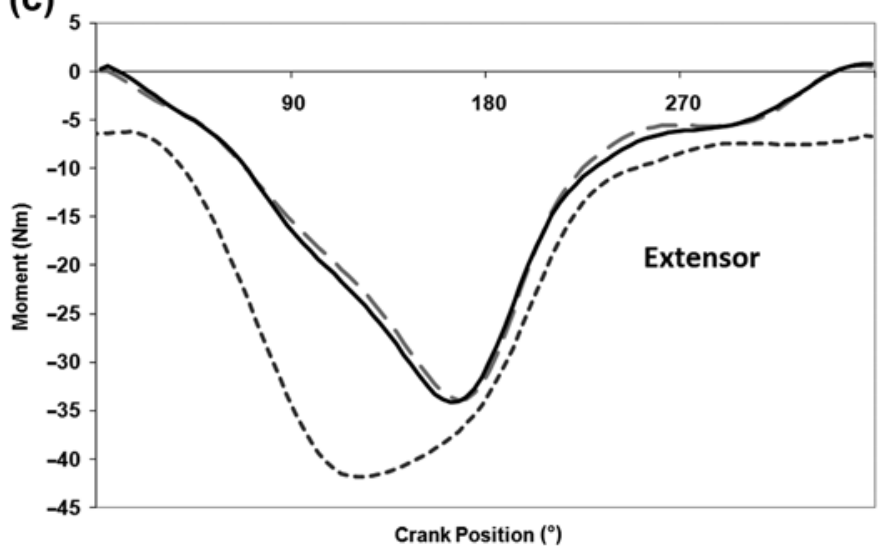

Figure 6.

(a) Hip, (b) knee, and (c) ankle joint moments for contralateral limb during control condition (short dashed line), amputated (AMP) limb during control condition (long dashed line), and AMP limb during CRANK condition (solid line). mance more than just focusing on symmetrical kinematics. Prosthetic kinetics may be directly measured in the clinic, and those devices are capable of providing real-time feedback to the clinician to improve prosthetic design [11-13]. This should be done while keeping in mind that the person is now operating with two very different limbs; therefore, an asymmetry may provide for the most functionally advantageous locomotion. The question now becomes whether there is an "optimal" amount of interlimb asymmetry? This will likely be different based on a multitude of factors, from limb length to integrity of the remaining neuromuscular system to prosthetic design. Future research may develop well-calibrated computer models based on experimental research to predict an optimal amount of asymmetry based off patient measurements, and then this could be verified by direct measurement during the fitting process. This knowledge would set quantifiable rehabilitation goals based on patient presentation and not what is considered "normal" based on the nondisabled population.

\section{ACKNOWLEDGMENTS}

\section{Author Contributions:}

Study concept and design: W. L. Childers, G. F. Kogler.

Acquisition of data: W. L. Childers, G. F. Kogler.

Analysis and interpretation of data: W. L. Childers, G. F. Kogler.

Drafting of manuscript: W. L. Childers.

Critical revision of manuscript for important intellectual content:

G. F. Kogler.

Statistical analysis: W. L. Childers.

Advising: G. F. Kogler.

Subject recruitment: W. L. Childers.

Financial Disclosures: The authors have declared that no competing interests exist.

Funding/Support: This material was based on work supported by the National Institutes of Health (grant T32 HD055180-01A1).

Additional Contributions: The authors gratefully acknowledge the volunteers that participated in this study; the staff of the School of Applied Physiology (Georgia Institute of Technology, Atlanta, Georgia) for their support throughout data collection; and Drs. Robert Gregor, Boris Prilutsky, Lena Ting, and Richard Nichols for their review of early versions of this article. We also thank Chris Hovorka and Arletha Willis, as well as Drs. Chad Duncan, Steven Chesbro, and Barney LeVeau, for always listening, supporting, and providing constructive feedback. Dr. Childers is no longer with the Georgia Institute of Technology.

Institutional Review: All volunteers read and signed an informed consent approved by the Institutional Review Board for the Georgia Institute of Technology before participating.

Participant Follow-Up: The authors plan to inform participants of the publication of this study. 


\section{REFERENCES}

1. Winter DA, Sienko SE. Biomechanics of below-knee amputee gait. J Biomech. 1988;21(5):361-67. [PMID:3417688] http://dx.doi.org/10.1016/0021-9290(88)90142-X

2. Sanderson D, Martin P. Lower extremity kinematic and kinetic adaptations in unilateral below-knee amputees during walking. Gait Posture. 1997;6:126-36.

http://dx.doi.org/10.1016/S0966-6362(97)01112-0

3. Chow DH, Holmes AD, Lee CK, Sin SW. The effect of prosthesis alignment on the symmetry of gait in subjects with unilateral transtibial amputation. Prosthet Orthot Int. 2006;30(2):114-28. [PMID:16990222] http://dx.doi.org/10.1080/03093640600568617

4. Silverman AK, Fey NP, Portillo A, Walden JG, Bosker G, Neptune RR. Compensatory mechanisms in below-knee amputee gait in response to increasing steady-state walking speeds. Gait Posture. 2008;28(4):602-9. [PMID:18514526] http://dx.doi.org/10.1016/j.gaitpost.2008.04.005

5. Childers WL, Kistenberg RS, Gregor RJ. Pedaling asymmetries in cyclists with unilateral transtibial amputation: Effect of prosthetic foot stiffness. J Appl Biomech. 2011; 27(4):314-21. [PMID:21896953]

6. Norvell DC, Czerniecki JM, Reiber GE, Maynard C, Pecoraro JA, Weiss NS. The prevalence of knee pain and symptomatic knee osteoarthritis among veteran traumatic amputees and nonamputees. Arch Phys Med Rehabil. 2005;86(3):487-93. [PMID:15759233]

http://dx.doi.org/10.1016/j.apmr.2004.04.034

7. Baker PA, Hewison SR. Gait recovery pattern of unilateral lower limb amputees during rehabilitation. Prosthet Orthot Int. 1990;14(2):80-84. [PMID:2235305]

8. Isakov E, Burger H, Krajnik J, Gregoric M, Marincek C. Influence of speed on gait parameters and on symmetry in trans-tibial amputees. Prosthet Orthot Int. 1996;20(3):153-58. [PMID:8985994]

9. Smith DG, Michael JW, Bowker JH. Chapter 30. Visual analysis of prosthetic gait. In: Atlas of amputations and limb deficiencies: Surgical, prosthetic, and rehabilitation principles. 3rd ed. Rosemont (IL): American Academy of Orthopedic Surgeons; 2004. p. 385-94.

10. Neumann ES, Yalamanchili K, Brink J, Lee JS. Transducerbased comparisons of the prosthetic feet used by transtibial amputees for different walking activities: A pilot study. Prosthet Orthot Int. 2012;36(2):203-16. [PMID:22344316] http://dx.doi.org/10.1177/0309364612436408

11. Boone DA, Kobayashi T, Chou TG, Arabian AK, Coleman KL, Orendurff MS, Zhang M. Influence of malalignment on socket reaction moments during gait in amputees with transtibial prostheses. Gait Posture. 2013;37(4):620-26. [PMID:23177920] http://dx.doi.org/10.1016/j.gaitpost.2012.10.002
12. Kobayashi T, Arabian AK, Orendurff MS, RosenbaumChou TG, Boone DA. Effect of alignment changes on socket reaction moments while walking in transtibial prostheses with energy storage and return feet. Clin Biomech (Bristol, Avon). 2014;29(1):47-56. [PMID:24315709] http://dx.doi.org/10.1016/j.clinbiomech.2013.11.005

13. Kobayashi T, Orendurff MS, Zhang M, Boone DA. Effect of alignment changes on sagittal and coronal socket reaction moment interactions in transtibial prostheses. J Biomech. 2013;46(7):1343-50. [PMID:23499228] http://dx.doi.org/10.1016/j.jbiomech.2013.01.026

14. Silver-Thorn MB, Steege JW, Childress DS. A review of prosthetic interface stress investigations. J Rehabil Res Dev. 1996;33(3):253-66. [PMID:8823673]

15. Nolan L, Wit A, Dudziñski K, Lees A, Lake M, Wychowañski $M$. Adjustments in gait symmetry with walking speed in transfemoral and trans-tibial amputees. Gait Posture. 2003;17(2): 142-51. [PMID:12633775] http://dx.doi.org/10.1016/S0966-6362(02)00066-8

16. Massaad F, Lejeune TM, Detrembleur C. The up and down bobbing of human walking: A compromise between muscle work and efficiency. J Physiol. 2007;582(Pt 2):789-99. [PMID:17463048] http://dx.doi.org/10.1113/jphysiol.2007.127969

17. Kuo AD, Donelan JM. Dynamic principles of gait and their clinical implications. Phys Ther. 2010;90(2):157-74. [PMID:20023002] http://dx.doi.org/10.2522/ptj.20090125

18. Komi PV. Chapter 4. Neuromechanics of the cycling task. In: Neuromuscular aspects of sports performance. Chichester (UK): Wiley-Blackwell; 2011. p. 52-77.

19. Zehr EP. Neural control of rhythmic human movement: The common core hypothesis. Exerc Sport Sci Rev. 2005; 33(1):54-60. [PMID:15640722]

20. Prilutsky BI. Coordination of two- and one-joint muscles: Functional consequences and implications for motor control. Motor Control. 2000;4(1):1-44. [PMID:10675807]

21. Prilutsky BI, Gregor RJ. Analysis of muscle coordination strategies in cycling. IEEE Trans Rehabil Eng. 2000;8(3): 362-70. [PMID:11001516] http://dx.doi.org/10.1109/86.867878

22. Neptune RR, Kautz SA, Zajac FE. Muscle contributions to specific biomechanical functions do not change in forward versus backward pedaling. J Biomech. 2000;33(2):155-64. [PMID:10653028] http://dx.doi.org/10.1016/S0021-9290(99)00150-5

23. Ting LH, Raasch CC, Brown DA, Kautz SA, Zajac FE. Sensorimotor state of the contralateral leg affects ipsilateral muscle coordination of pedaling. J Neurophysiol. 1998; 80(3):1341-51. [PMID:9744943]

24. Zajac FE. Understanding muscle coordination of the human leg with dynamical simulations. J Biomech. 2002; 
35(8):1011-18. [PMID:12126660]

http://dx.doi.org/10.1016/S0021-9290(02)00046-5

25. Heil DP, Derrick TR, Whittlesey S. The relationship between preferred and optimal positioning during submaximal cycle ergometry. Eur J Appl Physiol Occup Physiol. 1997;75(2):160-65. [PMID:9118982] http://dx.doi.org/10.1007/s004210050141

26. Childers WL, Kistenberg RS, Gregor RJ. The biomechanics of cycling with a transtibial amputation: Recommendations for prosthetic design and direction for future research. Prosthet Orthot Int. 2009;33(3):256-71. [PMID:19658015] http://dx.doi.org/10.1080/03093640903067234

27. Sanderson D. The influence of cadence and power output on asymmetry of force application during steady-rate cycling. J Hum Mov Stud. 1990;19:1-9.

28. Carpes FP, Rossato M, Faria IE, Bolli Mota C. Bilateral pedaling asymmetry during a simulated $40-\mathrm{km}$ cycling time-trial. J Sports Med Phys Fitness. 2007;47(1):51-57. [PMID:17369798]

29. Whaley MH, Brubaker PH, Otto RM, Armstrong LE. ACSM's guidelines for exercise testing and prescription. 7th ed. Philadelphia (PA): Lippincott Williams \& Wilkins; 2006.

30. Dempster WT. Space requirements of the seated operator: Geometrical, kinematic, and mechanical aspects of the body with special reference to the limbs. Wright Air Development Center technical report 55-159. Wright-Patterson Air Force Base (OH): Wright Air Development Center, Air Research and Development Command; 1955.

31. Broker J, Gregor R. A dual piezoelectric element force pedal for kinetic analysis of cycling. Int J Sport Biomech. 1990;6:394-404.

32. Wheeler J, Gregor R, Broker J. A dual piezoelectric bicycle pedal with multiple shoe pedal interface compatibility. Int $J$ Sport Biomech. 1992;8(3):251-58.

33. Pruitt AL, Matheny F. Andy Pruitt's complete medical guide for cyclists. Boulder (CO): VeloPress; 2004.

34. Childers WL, Perell-Gerson KL, Gregor RJ. Measurement of motion between the residual limb and the prosthetic socket during cycling. J Prosthet Orthot. 2012;24:19-24. http://dx.doi.org/10.1097/JPO.0b013e31824362e5

35. Neptune RR, Hull ML. Accuracy assessment of methods for determining hip movement in seated cycling. J Biomech. 1995;28(4):423-37. [PMID:7738051] http://dx.doi.org/10.1016/0021-9290(94)00080-N

36. Vaughan CL, Davis BL, O'Connor JC. Dynamics of human gait. 2nd ed. Cape Town (South Africa): Kiboho Publishers; 1999.

37. Smidt GL. Biomechanical analysis of knee flexion and extension. J Biomech. 1973;6(1):79-92. [PMID:4693870] http://dx.doi.org/10.1016/0021-9290(73)90040-7

38. Zatsiorsky V, Seluyanov V, Chugunova L. Methods of determining mass-inertial characteristics of human body segments. In: Chernyi GG, Regirer SA, editors. Contemporary problems of biomechanics. Moscow (Russia): Mir Publishers; 1990. p. 272-91.

39. de Leva P. Adjustments to Zatsiorsky-Seluyanov's segment inertia parameters. J Biomech. 1996;29(9):1223-30. [PMID:8872282]

http://dx.doi.org/10.1016/0021-9290(95)00178-6

40. Goldberg EJ, Requejo PS, Fowler EG. The effect of direct measurement versus cadaver estimates of anthropometry in the calculation of joint moments during above-knee prosthetic gait in pediatrics. J Biomech. 2008;41(3):695-700. [PMID:18031751] http://dx.doi.org/10.1016/j.jbiomech.2007.10.002

41. Smith JD, Martin PE. Effects of prosthetic mass distribution on metabolic costs and walking symmetry. J Appl Biomech. 2013;29(3):317-28. [PMID:22977207]

42. Broker JP, Gregor RJ. Mechanical energy management in cycling: Source relations and energy expenditure. Med Sci Sports Exerc. 1994;26(1):64-74. [PMID:8133741] http://dx.doi.org/10.1249/00005768-199401000-00012

43. Daly DJ, Cavanagh PR. Asymmetry in bicycle ergometer pedalling. Med Sci Sports. 1976;8(3):204-8. [PMID:979569]

44. Dingwell JB, Davis BL, Frazier DM. Use of an instrumented treadmill for real-time gait symmetry evaluation and feedback in normal and trans-tibial amputee subjects. Prosthet Orthot Int. 1996;20(2):101-10. [PMID:8876003]

45. Gregor RJ, Cavanagh PR, LaFortune M. Knee flexor moments during propulsion in cycling - a creative solution to Lombard's Paradox. J Biomech. 1985;18(5):307-16. [PMID:4008501] http://dx.doi.org/10.1016/0021-9290(85)90286-6

Submitted for publication November 8, 2013. Accepted in revised form May 8, 2014.

This article and any supplementary material should be cited as follows:

Childers WL, Kogler GF. Symmetrical kinematics does not imply symmetrical kinetics in people with transtibial amputation using cycling model. J Rehabil Res Dev. 2014;51(8):1243-54. http://dx.doi.org/10.1682/JRRD.2013.11.0241

ResearcherID/ORCID: W. Lee Childers, PhD, CP: M1781-2014; Géza F. Kogler, PhD, CO: M-1824-2014

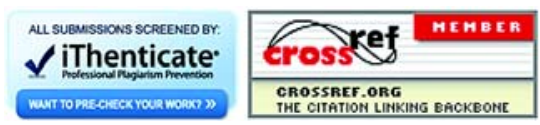


1254

JRRD, Volume 51, Number 8, 2014 
